\title{
Admisiones por bronquiolitis en 13 unidades de cuidados intensivos pediátricos del Uruguay: ¿es igual en Montevideo que en el interior?
}

Bronchiolitis admissions in 13 pediatric intensive care units in Uruguay: is it the same in Montevideo (capital city) and the provinces? Internações por bronquiolite em 13 unidades de terapia intensiva pediátrica do Uruguai: são iguais em Montevidéu e no Interior do país?

Luis Martínez-Arroyo¹, Franco Díaz-Rubio², Sebastián González-Dambrauskas³, Nicolás Monteverde-Fernández ${ }^{4}$, Alberto Serra ${ }^{5}$, Luis Eduardo Pedrozo Ortiz ${ }^{6}$, Lorena Soledad Menta Romano7, Luis Castro ${ }^{8}$, Pablo Vásquez-Hoyos ${ }^{9}$

\section{Resumen}

Objetivos: describir las características clínicas y epidemiológicas de niños admitidos por bronquiolitis en 13 unidades de cuidados intensivos pediátricos (UCIP) del Uruguay y comparar los resultados asistenciales finales entre UCIP de Montevideo (UM) y del interior del país (UI).

Material y método: estudio observacional retrospectivo multicéntrico de los registros ingresados a base de datos prospectiva de LARed Network. Se incluyeron niños mayores de 1 mes y menores de 2 años admitidos en el período 1 de mayo de 2017 y 30 de abril de 2019 con diagnóstico de bronquiolitis comunitaria. Se analizaron datos demográficos, clínicos, así como intervenciones y desenlaces al alta.

Resultados: se analizaron 666 casos. No se detectaron diferencias significativas de comorbilidades ni en el soporte respiratorio al ingreso. En UI los pacientes fueron derivados con más frecuencia desde otro hospital. La distancia y tiempo medio, así como el porcentaje de traslados mayor de 50 km, fue también mayor. En UI los pacientes tuvieron mayor gravedad clínica y gasométrica al ingreso. El perfil radiológico y etiológico fue similar. Virus respiratorio sincicial (VRS) aislado $>50 \%$. La indicación

1. Unidad de Cuidados Especiales Pediátricos y Neonatales. Corporación Médica de Paysandú (COMEPA), Paysandú, Uruguay. Red Colaborativa Pediátrica de Latinoamérica.

2. Red Colaborativa Pediátrica de Latinoamérica (LARed Network). Instituto de Ciencias e Innovación en Medicina (ICIM), Universidad del Desarrollo, Santiago, Chile. Unidad de Paciente Crítico, Hospital El Carmen de Maipú, Santiago, Chile.

3. Red Colaborativa Pediátrica de Latinoamérica (LARed Network) y Cuidados Intensivos Pediátricos Especializados (CIPe) Casa de Galicia, Montevideo, Uruguay.

4. Red Colaborativa Pediátrica de Latinoamérica (LARed Network) y Cuidados Intensivos Pediátricos y Neonatales (CINP), Médica Uruguaya, Montevideo, Uruguay.

5. Red Colaborativa Pediátrica de Latinoamérica (LARed Network) y Cuidados Intensivos Pediátricos Especializados (CIPe) Casa de Galicia, Montevideo, Uruguay.

6. Red Colaborativa Pediátrica de Latinoamérica (LARed Network). Unidad de Cuidados Intensivos de Niños y Recién Nacidos del Hospital Regional Salto. ASSE. Salto. Uruguay.

7. Red Colaborativa Pediátrica de Latinoamérica (LARed Network). Área de Vigilancia Pediátrica. Hospital Regional de Tacuarembó. Uruguay.

8. Red Colaborativa Pediátrica de Latinoamérica (LARed Network). Unidad de Cuidados Intensivos Neonatales y Pediátricos de CAMDEL. Minas, Lavalleja. Uruguay.

9. Red Colaborativa Pediátrica de Latinoamérica (LARed Network), Unidad de Cuidado Intensivo Pediátrico. Sociedad de Cirugía Hospital de San José, Bogotá. Departamento de Pediatría, Fundación Universitaria de Ciencias de la Salud, Bogotá. Departamento de Pediatría, Universidad Nacional de Colombia, Bogotá, Colombia.

En nombre de la LARed Network.

Sin financiación externa.

Los autores declaran no tener conflictos de intereses.

Colaboradores: 13 Unidades de Cuidados Intensivos Pediátricos del Uruguay.

Correspondencia: Luis Martínez-Arroyo. Correo electrónico: luismartinezarroyo33@gmail.com

Recibido: 2/9/2020

Aprobado: $13 / 1 / 21$

Attribution-NonCommercial 4.0 International (CC BY-NC 4.0) 
global de corticoides superó el $25 \%$ y el de broncodilatadores el $85 \%$. La prescripción de antibióticos y adrenalina nebulizada fue mayor en UI. La cánula nasal de alto flujo (CNAF) fue globalmente el método de soporte respiratorio más utilizado, aunque se observó un mayor uso de ventilación mecánica invasiva (VMI) y CPAP en UI ( $47 \%$ vs $28 \%$ en UM). No hubo diferencias en el número de complicaciones por VMI o ventilación no invasiva, ni en el uso de terapias de rescate. Tampoco se notaron diferencias significativas en la duración de la estadía en UCIP, ni en la mortalidad absoluta y ajustada, y hubo un solo caso de nueva morbilidad.

Conclusiones: los niños admitidos en UI tuvieron mayor gravedad al ingreso y más factores de riesgo relacionados con mal pronóstico en el traslado, recibiendo más antibióticos y soporte invasivo que aquellos ingresados en UM. El CNAF fue el tipo de soporte respiratorio más utilizado en el país. Se detectó alto porcentaje de prescripción de terapias no recomendadas, como broncodilatadores y corticoides. La mortalidad y complicaciones fueron bajas, así como la generación de morbilidad residual.

Palabras clave: Bronquiolitis

Unidades de Cuidado intensivo pediátrico

Ventilación no invasiva

Morbilidad

Niño

Cánula nasal de alto flujo

Key words: Bronchiolitis

Intensive care units, pediatric

Noninvasive ventilation

Morbidity

Child

High flow nasal cannula

\section{Introducción}

La bronquiolitis es una enfermedad viral aguda que afecta a la vía aérea inferior de niños menores de 2 años y constituye la principal causa de ingreso hospitalario y en las Unidades de Cuidados Intensivos Pediátricos (UCIP) durante los meses de invierno ${ }^{(1,2)}$. Tiene varias definiciones operativas, con gran variabilidad entre centros y países $^{(3)}$, siendo la más aceptada que es el primer episodio de sibilancias o subcrepitantes asociadas a una infección viral en niños menores de 24 meses $^{(4-6)}$. El principal agente causal es el virus respiratorio sincitial (VRS). Se han descrito varias comorbilidades que se asocian a una evolución más grave como la prematurez y enfermedades previas neuromusculares o respiratorias, entre otras. El tratamiento de la bronquiolitis es de soporte, sin que exista una clara evidencia de que otras medidas terapéuticas, como broncodilatadores, adrenalina, suero salino hipertónico, corticoides o inhibidores de los leucotrienos, sean eficaces en su tratamiento; no obstante, existe una amplia variabilidad en la aplicación de distintas intervenciones bajo la consideración de que

existen diferentes "fenotipos" asociados a las mismas a nivel mundial ${ }^{(4-7)}$.

Los pacientes internados suelen requerir apoyo respiratorio con oxigenoterapia convencional o con cánulas nasales de alto flujo (CNAF) cuya aplicación se ha difundido cada vez más a las salas de internación gene$\mathrm{ral}^{(8)}$. Sin embargo, a pesar de estas medidas, un porcentaje de estos pacientes presentan una insuficiencia respiratoria u otro tipo de complicación que amerita su ingreso en UCIP. El fenómeno de variabilidad de atención e indicación de los distintos tipos de tratamientos y soporte utilizados es también frecuente, así como en la evolución y pronóstico de las mismas ${ }^{(4,9)}$.

La Red Colaborativa Latinoamericana de Cuidados Intensivos Pediátricos (LARed Network) es una red profesional que creó un registro multinacional para una amplia gama de variables de los pacientes ingresados por fallo respiratorio en 35 UCIP de 8 países de Latinoamérica con el objetivo de compartir resultados y lograr una mejora continua de la calidad basada en la colaboración. En nuestro país son 13 las unidades que participan, 7 pertenecientes a Montevideo y 6 radicadas en el interior del país ${ }^{(10)}$. 
Hasta nuestro conocimiento no existen trabajos que aborden a escala nacional y multicéntrica la caracterización de los niños ingresados por bronquiolitis grave en UCIP de Uruguay. Tampoco encontramos descripciones comparativas de desenlaces asistenciales de niños tratados en centros del interior del país con respecto a aquellos asistidos en la capital nacional. El alto impacto asistencial que tiene la bronquiolitis en nuestro país amerita que esta brecha de investigación sea completada.

\section{Objetivos}

Describir las características clínicas y epidemiológicas de niños admitidos por bronquiolitis en 13 Unidades de Cuidados Intensivos Pediátricos (UCIP) del Uruguay durante los dos últimos años y realizar un análisis comparativo de los resultados asistenciales finales entre UCIP de Montevideo (UM) y del interior del país (UI).

\section{Material y método}

Estudio observacional retrospectivo multicéntrico de los registros ingresados de forma prospectiva en la base de datos de la Red Latinoamericana de Cuidados Intensivos Pediátricos (LARed Network) de pacientes mayores de 1 mes y menores de 2 años internados en las UCIP del país perteneciente a LARed Network en el período 1 de mayo de 2017 y 30 de abril de 2019 con diagnóstico, por el médico tratante, de bronquiolitis comunitaria (independiente de si cumplían o no criterios clásicos de bronquiolitis o si presentaban eventos sibilantes previos). Todos los centros cuentan con aval institucional. Se utilizó un software seguro para la captura y manejo de datos (RedCAPTM Research Electronic Data Capture de la Universidad de Vanderbilt, USA) para su uso estrictamente epidemiológico ${ }^{(1)}$.

Se analizaron las características poblacionales: edad, sexo, procedencia (urbana, suburbana, rural y externa o interna) y las comorbilidades previas incluidas respiratorias, como sibilancias (asma, sibilantes recurrentes, hiperreactividad bronquial y eventos previos de enfermedades de vía aérea pequeña). En los pacientes trasladados se describe el tiempo y la distancia (continua y categórica). Se documentó el tipo de soporte con el paciente que llega a la unidad, la gravedad al ingreso (Pediatric Index Mortality-3, PIM-3) de riesgo de mortalidad), escalas de gravedad respiratoria $\left(\mathrm{Wang}^{(12)}, \mathrm{Liu}^{(13)}\right.$ y Woods ${ }^{(14)}$, valores de $\mathrm{SpO}_{2} \mathrm{FiO}_{2}, \mathrm{PaO}_{2} / \mathrm{FiO}_{2}$, pH y $\mathrm{pCO}_{2}$, estudios radiológicos y virales, tipo de soporte respiratorio utilizado en la unidad (inicial, global y máximo) y complicaciones derivadas, tratamientos utilizados, aparición de nuevas morbilidades (medidas con el aumento del Funcional Status Score $\left[\right.$ FSS ${ }^{(15)}$ en más de tres puntos al egreso en relación con el ingreso) y mortalidad con su razón estandarizada de mortalidad (REM) (calculada como la razón entre la mortalidad observada y la calculada a través del puntaje PIM-3).

Para su análisis se emplearon proporciones para variables cualitativas, y medianas y rangos intercuartiles para variables numéricas. Para el análisis de variables entre poblaciones se utilizó la prueba de chi cuadrado (con análisis del test de Fisher o de Pearson según el número de eventos por celda) para variables cualitativas y test de Mann-Whitney o Kruskall-Wallis para variables cuantitativas. Se consideró significación estadística un valor de $\mathrm{p}<0,05$. Se utilizó para su análisis el paquete estadístico STATA en su versión 13.

La participación en el registro contemporáneo de LARed incluye la autorización institucional y de comités de ética de todos los centros participantes para el volcado de datos anónimos, de identificados y permite los subanálisis de resultados asistenciales en el marco de la iniciativa continental de mejoría de la calidad asistencial de centros miembro, como este trabajo. Para garantizar la seguridad de dicha base de datos, LARed utiliza Redcap, un software seguro y avalado globalmente para estudios internacionales multicéntricos, y también avalado por centros participantes (RedCAPTM Research Electronic Data Capture de la Universidad de Vanderbilt, USA) para su uso estrictamente epidemiológico ${ }^{(10)}$. También este proyecto cuenta con la aprobación internacional del Comité de Ética Médica de unos de los centros de referencia de LA Red.

\section{Resultados}

Durante el período de observación, 666 casos cumplieron con los criterios de inclusión y exclusión. En relación con las características poblacionales, no hubo diferencias en grupos etarios y sexo, con predominio global del sexo masculino (relación 1,7/1), y una mayor proporción de etnia caucásica en la población del interior; la procedencia fue mayoritariamente urbana en ambas regiones, con una proporción mayor de origen suburbano en Montevideo en relación con la rural. En la capital casi la mitad de los pacientes ingresaron del servicio de urgencia, mientras que en el interior lo hacían desde el servicio de pediatría y más de $20 \%$ desde otro hospital (tabla 1).

El porcentaje de pacientes de UI que requirieron traslados mayores a $50 \mathrm{~km}$ fue significativamente mayor en relación con UM, así como el promedio de duración del traslado (tabla 1). No hubo diferencias en el tipo de soporte respiratorio con el que los pacientes llegaban a la unidad, siendo la cánula nasal el más frecuente (más de 75\%); el porcentaje de pacientes intubados antes de la admisión fue bajo y similar en ambos grupos (menos de $3 \%$ ) (tabla 5). 
Tabla 1. Características demográficas. Comparación de región de Uruguay.

\begin{tabular}{|c|c|c|c|c|c|}
\hline \multirow[t]{2}{*}{ Variable } & \multicolumn{2}{|c|}{ Interior (UI) } & \multicolumn{2}{|c|}{ Montevideo (UM) } & \multirow[t]{2}{*}{$P^{*}$} \\
\hline & \multicolumn{2}{|c|}{$N=184$} & \multicolumn{2}{|c|}{$N=482$} & \\
\hline Edad (meses), med riq & 4,0 & 5,5 & 4,4 & 6,3 & 0,173 \\
\hline $1 \mathrm{~m}-6 \mathrm{~m}$ & 120 & $65 \%$ & 293 & $61 \%$ & 0,546 \\
\hline $6-12 m$ & 44 & $24 \%$ & 134 & $28 \%$ & \\
\hline$>12 \mathrm{~m}$ & 20 & $11 \%$ & 55 & $11 \%$ & \\
\hline
\end{tabular}

Sexo, $n \%$

$\begin{array}{lcccc}\text { Femenino } & 71 & 39 \% & 176 & 37 \% \\ \text { Masculino } & 113 & 61 \% & 305 & 63 \% \\ \text { Indeterminado } & - & - & 1 & 0,2 \%\end{array}$

\begin{tabular}{lccccc}
\hline Etnia, $n \%$ & & & & & \\
Caucásica & 173 & $94 \%$ & 420 & $87 \%$ & 0,026 \\
Mestiza & 11 & $6 \%$ & 56 & $12 \%$ & \\
Otra & - & - & 6 & $1 \%$ & \\
\hline
\end{tabular}

Zona, $n \%$

\begin{tabular}{lrrrrr} 
Urbana & 165 & $90 \%$ & 386 & $80 \%$ & 0,000 \\
Suburbana & 9 & $5 \%$ & 90 & $19 \%$ & \\
Rural & 10 & $5 \%$ & 6 & $1 \%$ & \\
\hline
\end{tabular}

Procedencia, $n \%$ (**)

\begin{tabular}{|c|c|c|c|c|c|}
\hline Urgencias & 24 & $13 \%$ & 236 & $49 \%$ & 0,004 \\
\hline Pediatría general & 120 & $65 \%$ & 205 & $43 \%$ & 0,022 \\
\hline Otra UCI & - & - & 1 & $0.2 \%$ & 0,432 \\
\hline Otro hospital & 39 & $21 \%$ & 36 & $8 \%$ & 0,025 \\
\hline Otros & 1 & $1 \%$ & 4 & $1 \%$ & 0,536 \\
\hline Traslados externos & \multicolumn{2}{|c|}{$N=110$} & \multicolumn{2}{|c|}{$N=207$} & \\
\hline Tiempo en horas, med RIQ & 0,7 & 1,5 & 0,5 & 0,7 & 0,023 \\
\hline Distancia km, med RIQ & 26 & 150 & 10 & 19 & 0,585 \\
\hline Distancia mayor de $50 \mathrm{~km}$ & 54 & $49 \%$ & 40 & $19 \%$ & 0,000 \\
\hline
\end{tabular}

* Comparaciones por medio de pruebas de chi cuadrado (Fisher para menos de cinco eventos por celda o Pearson para las demás) o pruebas no paramétricas de Mann-Whitney o Kruskal Wallis, según el tipo de variable. Comparación de medianas por prueba no paramétrica de Wilcoxon; $\mathrm{p}<0,05$ se considera estadísticamente significativo.

** Para hacer estas comparaciones de subgrupo se utilizaron los residuos ajustados de la prueba de Pearson. Para la interpretación de la p en este caso se debe hacer un ajuste de Bonferroni para quitar el efecto de las comparaciones múltiples $[\mathrm{p}<0,05 / \mathrm{n}=0,01 ;(\mathrm{n}=5)]$.

$\mathrm{n} \%$ : número absoluto y porcentaje columna; med riq: mediana y rango intercuartílico; UCI: unidad de cuidado intensivo.

En relación con las comorbilidades hay un predominio de los tipos no respiratorios en ambos grupos. De forma llamativa hay una mayor proporción de displasia broncopulmonar en las UI (52\%) y de sibilantes previos en las UM $(82 \%)$. La principal comorbilidad no respira- toria fue la prematurez, sin diferencias entre ambas poblaciones (tabla 2).

Los pacientes de las UI tuvieron indicadores de mayor gravedad a su ingreso, reflejados por una probabilidad mayor de muerte por el score PIM-3, así como por 
Tabla 2. Frecuencias y tipos de comorbilidades. Comparación de región de Uruguay.

\begin{tabular}{|c|c|c|c|c|c|}
\hline \multirow[t]{3}{*}{ Variable } & \multirow{2}{*}{\multicolumn{2}{|c|}{$\begin{array}{l}\text { Interior (UI) } \\
\qquad N=184\end{array}$}} & \multirow{2}{*}{\multicolumn{2}{|c|}{$\begin{array}{c}\text { Montevideo (UM) } \\
\qquad N=482\end{array}$}} & \multirow[t]{3}{*}{$p^{* *}$} \\
\hline & & & & & \\
\hline & $n$ & $\%$ & \multicolumn{2}{|r|}{$\%$} & \\
\hline Comorbilidades & 52 & $28 \%$ & 170 & $35 \%$ & 0,086 \\
\hline Comorbilidades respiratorias & 31 & $17 \%$ & 96 & $20 \%$ & 0,367 \\
\hline Displasia broncopulmonar & 16 & $52 \%$ & 11 & $12 \%$ & 0,000 \\
\hline Sibilantes* & 12 & $39 \%$ & 79 & $82 \%$ & 0,000 \\
\hline Otros & 3 & $10 \%$ & 8 & $8 \%$ & 0,727 \\
\hline Otras comorbilidades & 42 & $23 \%$ & 104 & $22 \%$ & 0,727 \\
\hline Cardiopatías & 7 & $17 \%$ & 14 & $14 \%$ & 0,617 \\
\hline Prematuro & 24 & $57 \%$ & 56 & $54 \%$ & 0,717 \\
\hline Genopatía & 5 & $12 \%$ & 20 & $19 \%$ & 0,340 \\
\hline Déficit neurológico & 4 & $10 \%$ & 13 & $12 \%$ & 0,779 \\
\hline Otros & 7 & $17 \%$ & 14 & $14 \%$ & 0,617 \\
\hline Oxígeno en casa & - & - & 4 & $1 \%$ & \\
\hline
\end{tabular}

un mayor puntaje en las escalas de Wang, Liu y Woods a su admisión en la unidad. La SpO2FiO2 media tuvo valores significativamente menores y el porcentaje de pacientes con un valor de pCO2 mayor a $50 \mathrm{~mm} \mathrm{Hg}$ también fue mayor en las UI. No hubo diferencias significativas en los valores medios de $\mathrm{PaO}_{2} / \mathrm{FiO}_{2}, \mathrm{pCO}_{2}$ y pH entre ambas poblaciones (tabla 3 ).

Los hallazgos radiológicos fueron similares en ambas poblaciones, salvo un mayor diagnóstico de consolidación en la UI. No existieron diferencias en la etiología viral, predominando el VRS en ambos grupos y con una identificación de coinfección viral baja (menor de 3\%) en ambos grupos; la única diferencia fue una mayor proporción de sospecha de sobreinfección bacteriana en las UM (8\%) en relación con las UI (3\%) (tabla 4).

Hubo un mayor uso de antibióticos en las UI (62\%) en relación con las UM (42\%), así como de suero salino hipertónico (35\% en UI vs $8 \%$ en UM). El empleo de broncodilatadores fue muy frecuente en ambas poblaciones (más del 85\%), seguido de los corticoides (más del $25 \%$ ) y con una utilización marginal de la adrenalina nebulizada (menos del 7\%), sin diferencias en ninguno de los tres casos entre los grupos (tabla 4).
La CNAF fue utilizada como soporte respiratorio inicial de una forma significativamente mayor en las UM $(87 \%)$ en relación con las UI (63\%). Por el contrario, el uso de CPAP $(10,3 \%)$ y la ventilación mecánica invasiva (VMI) (13\%) fue usado más frecuentemente en la admisión en las UI en relación con las UM $(2 \%$ y $6 \%$, respectivamente). Este predominio en la utilización del $\mathrm{CNAF}$ en relación con las modalidades de soporte respiratorio (CPAP, BPAP y VMI) en las UM respecto a las del interior se mantuvo también durante la internación, tanto en su uso global como en el soporte máximo que llegó a necesitar el paciente (tabla 5).

La tasa de complicaciones de la VMI fue similar en ambas poblaciones (en torno a 21\%), sin diferencias significativas en cuanto al tipo de éstas, siendo los principales problemas el fallo en el destete y el síndrome de abstinencia; el porcentaje de neumotórax fue similar (menor a 3\%). Hubo pocas complicaciones relacionadas a la ventilación mecánica no invasiva (VMNI), principalmente síndrome de abstinencia, sin diferencia entre grupos, y no se registró ninguna fuga aérea. Tampoco existieron diferencias en el uso de terapias de rescate ventilatorias (ventilación de alta frecuencia [VAFO]), uso de 
Tabla 3. Escalas de gravedad e índices gasométricos y de oxigenación al ingreso. Comparación de región de Uruguay.

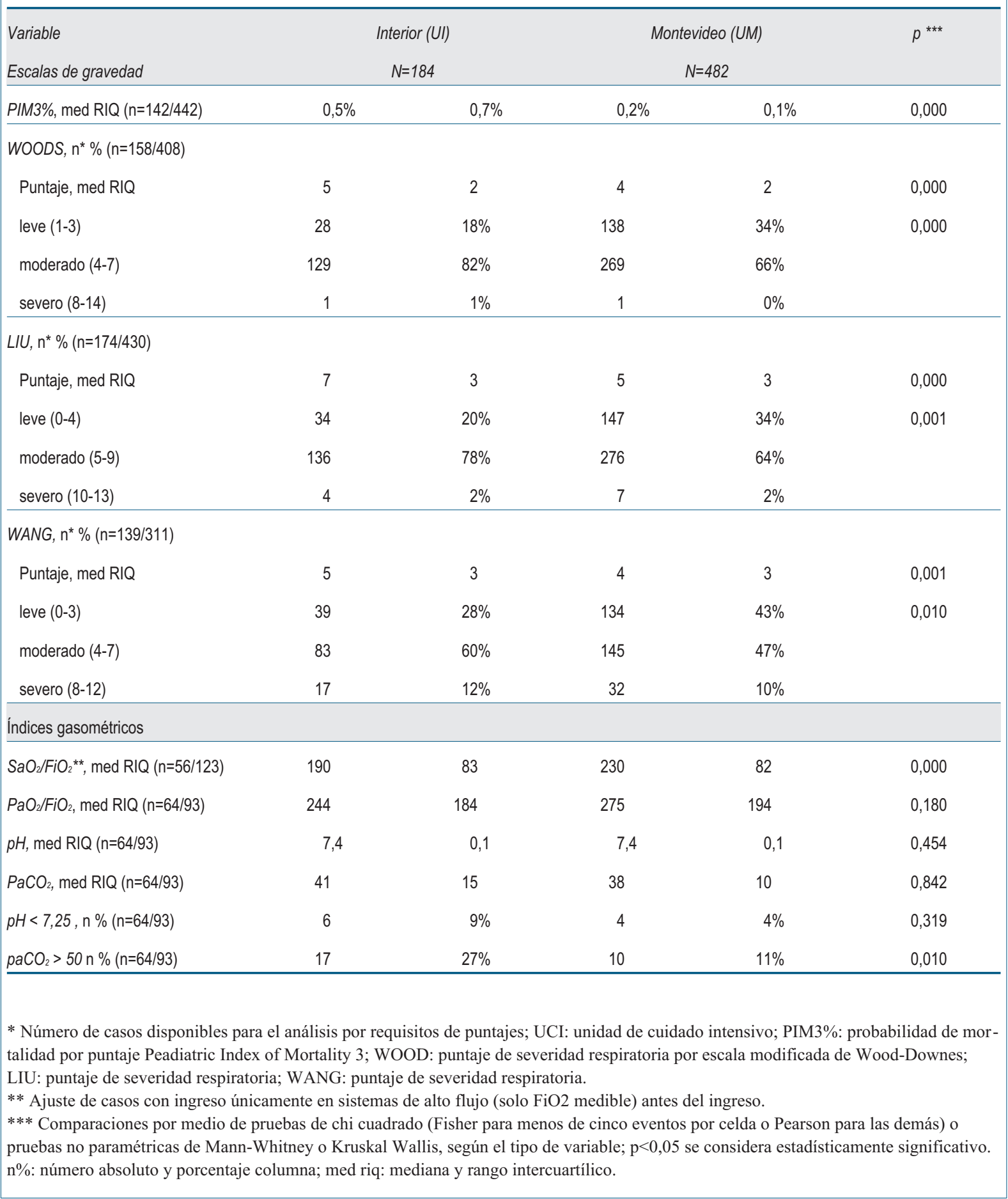

surfactante, uso de bloqueantes neuromusculares, óxido nítrico inhalado, ventilación en decúbito prono), utilizándose algunas de éstas (bloqueantes musculares y el decúbito prono) en menos de $7 \%$ (tabla 6 ).
La mediana de estadía fue similar en ambos grupos (4,9 días [RIQ 5,6] en UI vs 5,0 días [RIQ 3,4] en UM $\mathrm{p}=0,678)$, sin diferencias significativas en la mortalidad ( $1 \%$ en UI y $0 \%$ en UM). La REM fue de 0,42 (IC 95\%: 
Tabla 4. Estudios radiológicos y virales. Tipos de tratamientos utilizados. Comparación de región Uruguay.

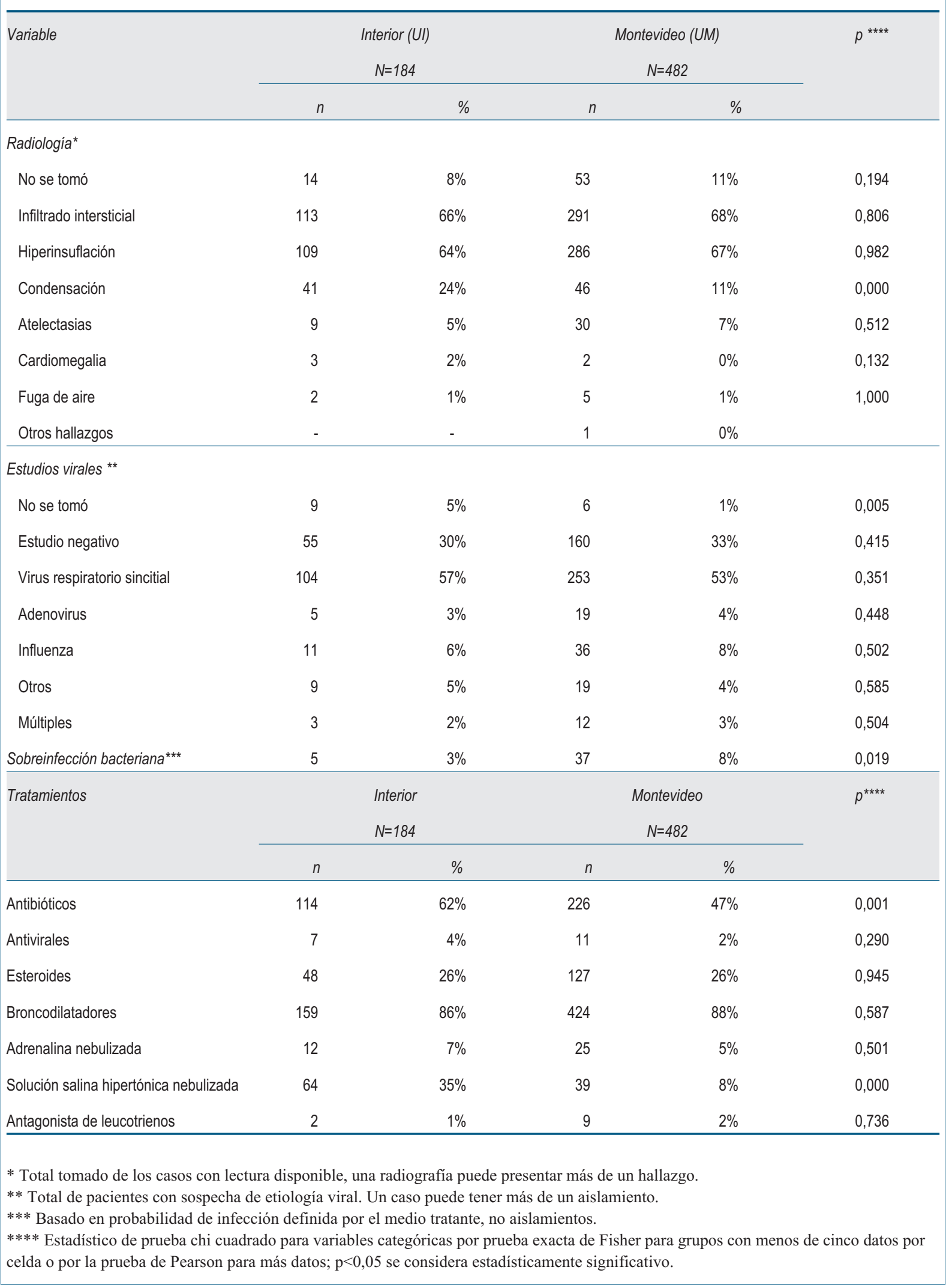


Tabla 5. Soporte ventilatorio en unidad. Comparación de región de Uruguay.

\begin{tabular}{lccccc}
\hline \multicolumn{1}{c}{ Variable } & \multicolumn{2}{c}{ Interior (UI) } & \multicolumn{2}{c}{ Montevideo (UM) } & $p^{* *}$ \\
\hline Soporte a la llegada, $n \%^{*}$ & \multicolumn{2}{c}{$N=184$} & \multicolumn{2}{c}{$N=482$} & 0,683 \\
VMl & 5 & $3 \%$ & 12 & $3 \%$ & 0,107 \\
BIPAP & - & - & 7 & $2 \%$ & 0,119 \\
CPAP & 9 & $5 \%$ & 7 & $18 \%$ & 0,173 \\
CNAF & 19 & $10 \%$ & 87 & $75 \%$ & $1 \%$ \\
Oxigenoterapia & 151 & $82 \%$ & 363 & 6 &
\end{tabular}

Soporte UCIP inicial, $n \%$

\begin{tabular}{lccccc} 
VMI & 23 & $13 \%$ & 28 & $6 \%$ & 0,089 \\
BIPAP & 8 & $4 \%$ & 11 & $2 \%$ & 0,232 \\
CPAP & 19 & $10 \%$ & 8 & $2 \%$ & 0,024 \\
CNAF & 115 & $63 \%$ & 419 & $87 \%$ & 0,008 \\
Oxigenoterapia & 19 & $10 \%$ & 15 & $3 \%$ & 0,052 \\
\hline
\end{tabular}

Soporte máximo, $n \%$

\begin{tabular}{|c|c|c|c|c|c|}
\hline VMI & 57 & $31 \%$ & 89 & $19 \%$ & 0,062 \\
\hline BIPAP & 8 & $4 \%$ & 25 & $5 \%$ & 0,084 \\
\hline CPAP & 11 & $6 \%$ & 8 & $2 \%$ & 0,164 \\
\hline CNAF & 95 & $52 \%$ & 345 & $72 \%$ & 0,027 \\
\hline Ninguno $\left(\mathrm{O}_{2}\right.$ /ambiente $)$ & 13 & $7 \%$ & 15 & $3 \%$ & 0,132 \\
\hline \multicolumn{6}{|l|}{ Soporte en algún momento* $n$ \% } \\
\hline VMI & 57 & $31 \%$ & 89 & $19 \%$ & 0,062 \\
\hline BIPAP & 17 & $9 \%$ & 52 & $11 \%$ & 0,557 \\
\hline CPAP & 30 & $16 \%$ & 44 & $9 \%$ & 0,008 \\
\hline CNAF & 151 & $82 \%$ & 450 & $93 \%$ & 0,000 \\
\hline Total de eventos de soporte ${ }^{*}, n \%$ & \multicolumn{2}{|c|}{$\mathrm{N}=386$} & \multicolumn{2}{|c|}{$N=889$} & \\
\hline VMI & 66 & $17 \%$ & 97 & $11 \%$ & 0,081 \\
\hline BIPAP & 18 & $5 \%$ & 55 & $6 \%$ & 0,300 \\
\hline CPAP & 38 & $10 \%$ & 49 & $6 \%$ & 0,093 \\
\hline CNAF & 172 & $45 \%$ & 513 & $58 \%$ & 0,038 \\
\hline Oxigenoterapia & 92 & $24 \%$ & 175 & $20 \%$ & 0,196 \\
\hline
\end{tabular}

* Puede suceder más de un evento de soporte ventilatorio por ingreso.

* Estadístico de prueba chi cuadrado para variables categóricas por prueba de Pearson; $\mathrm{p}<0,05$ se considera estadísticamente significativo. CNAF: cánula nasal de alto flujo; CPAP: presión positiva continua en la vía aérea; BIPAP: ventilación mecánica no invasiva (presión positiva de dos niveles); VMI: ventilación mecánica invasiva; UCI: unidad de cuidado intensivo. 


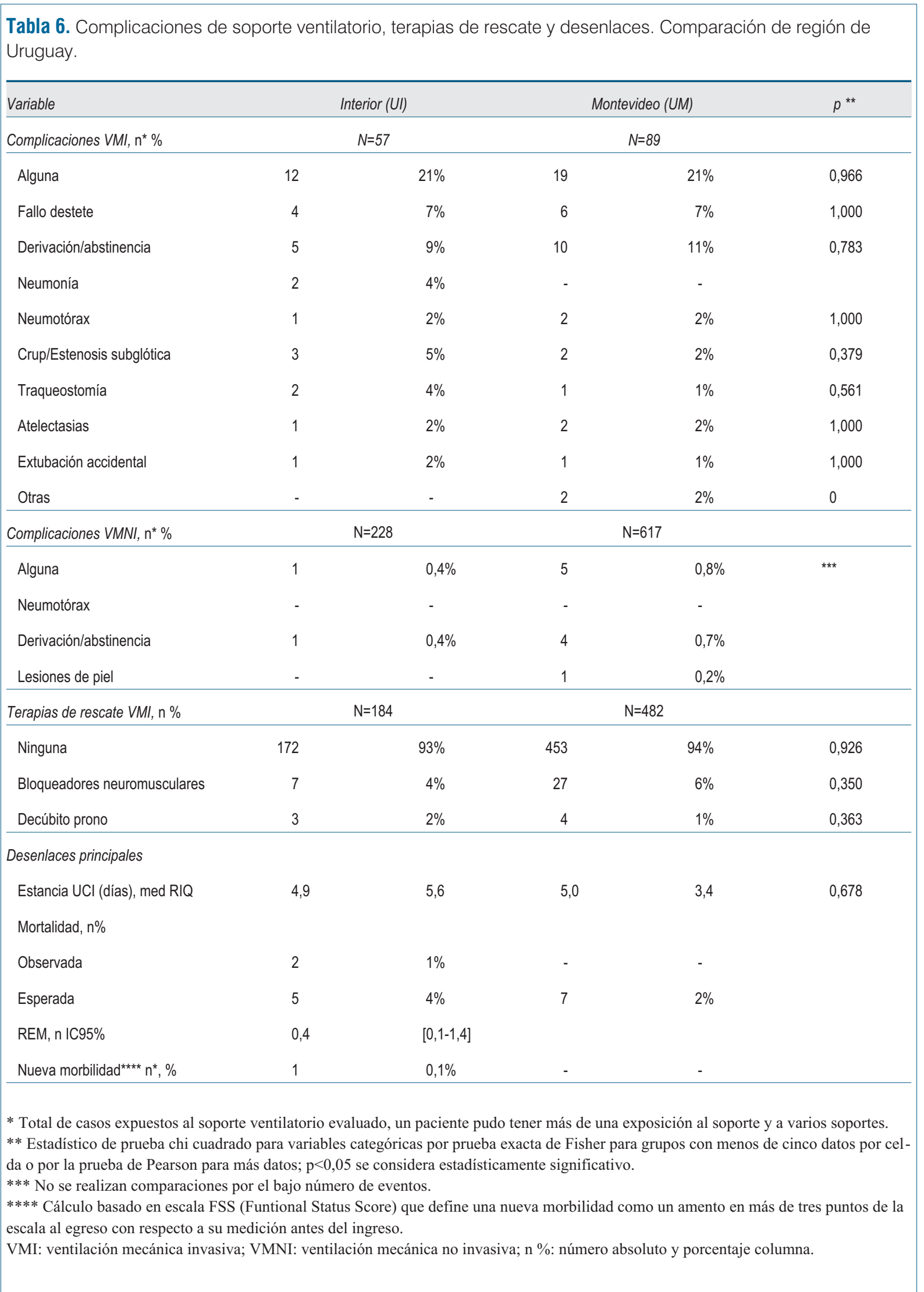


$0,07-1,35)$ en las UI y de 0 en las UM. Solo hubo un caso de nueva morbilidad (aumento de más de tres puntos al egreso del FSS) en las UI y ninguno en las UM (tabla 6).

\section{Discusión}

Hasta nuestro conocimiento, este estudio analiza la cohorte de niños con bronquiolitis internados en UCIP nacionales más grande a la fecha. Los resultados muestran importantes diferencias en el escenario clínico y manejo de esta heterogénea entidad entre unidades del interior y la capital del país.

Los pacientes son menores de 6 meses en más del $60 \%$ de los casos, con un predominio masculino y de procedencia en su mayoría urbana; algunos elementos encontrados, como un mayor porcentaje de población rural y de traslados desde otros hospitales en las UI, pueden explicar algunos de los indicadores asociados a una mayor gravedad al ingreso en estos centros debido a la menor accesibilidad al sistema de salud en el medio rural y al impacto que tiene el transporte de un paciente con insuficiencia respiratoria aguda, sobre todo si el trayecto supera los $50 \mathrm{~km}$, como se objetivó en un trabajo previo de LARed y en este estudio, donde casi el 50\% de los traslados superaba esta distancia ${ }^{(16,17)}$.

La presencia de comorbilidades en ambas poblaciones fue similar a la reportada en la mayoría de los estudios, siendo más frecuentes las vinculadas a aspectos no respiratorios, en su mayoría prematurez, aunque la presencia de cardiopatías, genopatías y trastornos neurológicos no son nada infrecuentes ${ }^{(1,9,18,19,20,-21)}$. En relación con las comorbilidades respiratorias existe una diferencia en los porcentajes reportados de displasia bronco-pulmonar y sibilante previo entre UI y UM, aunque pensamos que puede deberse a distintos conceptos en la definición operacional de la propia base de datos ${ }^{(3)}$. No se observaron pacientes con oxígeno domiciliario en las UI y solo $1 \%$ en las UM. Al comparar con otros trabajos algunas morbilidades, como la displasia broncopulmonar y la prematurez, son algo más elevadas que en trabajos nacionales e internacionales previos ${ }^{(1,9,18-22)}$.

La mayor gravedad de la población de las UI al ingreso se pone de manifiesto tanto en escalas pronósticas de mortalidad al ingreso (PIM-3) ${ }^{(23)}$, como en puntajes más elevados en escalas de valoración respiratoria. Si bien en nuestro país la escala utilizada en la valoración de la gravedad de las bronquiolitis es el puntaje de TAL modificado ${ }^{(24,25)}$, nuestra base de datos, al tratarse de una red multinacional y que valora otras patologías respiratorias ingresadas en las UCIP, además de la bronquiolitis, cuenta con otras escalas validadas a tal fin. El puntaje de Wang ha sido evaluado y validado para su uso en bronquiolitis $^{(12)}$, mientras que la escala de $\mathrm{Liu}^{(13)}$ fue diseñada para evaluar la dificultad respiratoria en asma y bronquiolitis entre diferentes proveedores de servicios de salud; la escala de Wood-Downes es ampliamente conocida y utilizada en la evaluación de la crisis asmáti$\mathrm{ca}^{(14)} \mathrm{y}$ ha sido validada en la bronquiolitis ${ }^{(26)}$. En los tres casos tanto el puntaje promedio como el porcentaje de pacientes clasificados en dichas escalas como moderados/severos, fueron significativamente mayores en las UI, lo que refuerza la noción de mayor gravedad respiratoria.

El porcentaje de pacientes clasificados como más graves en las escalas de Wood-Downes y Liu fue llamativamente bajo, lo que no ocurrió con el puntaje de Wang, en donde más del 10\% de los ingresos fueron catalogados como severos. Al contrario de lo que podría pensarse al tratarse de pacientes ingresados en UCIP, los porcentajes catalogados como leves son mayores que los severos, sobre todo en las UM con porcentajes mayores a 30\%. Este aspecto podría verse relacionado con varios factores, como puede ser la presencia de otras causas no vinculadas a la gravedad respiratoria como determinantes del ingreso en UCIP, la no disponibilidad de soporte con CNAF en urgencias o sala de pediatría en algunas instituciones u otros factores vinculados a la estructura y funcionamiento. Esta variabilidad entre centros, regiones o países está documentada por nuestro grupo y otras series internacionales ${ }^{(9,20,21,27)}$ y es un claro punto detectado como mejorable en el sistema sanitario.

Esta mayor gravedad de la población ingresada en las UI también se vio corroborada por el hecho de que la $\mathrm{SpO}_{2} / \mathrm{FiO}_{2}$ promedio era más baja (190) en UI que en UM (230), valores que algunas guías recomiendan para la aplicación de BIPAP o CNAF. También el porcentaje de pacientes con valores de $\mathrm{PCO}_{2}$ mayores a $50 \mathrm{~mm} \mathrm{Hg}$, que algunos autores identifican como indicador de mayor riesgo de fracaso de soporte con OAF. Si bien la $\mathrm{PaO}_{2} / \mathrm{FiO}_{2}$ fue algo menor en las UI, no fue estadísticamente significativa, así como tampoco los valores promedios de $\mathrm{pH}$ y de $\mathrm{PCO}_{2}$. Cabe destacar el uso cada vez más extendido de la $\mathrm{SpO}_{2} / \mathrm{FiO}_{2}$ como método de valorar la hipoxemia de la insuficiencia respiratoria, dada la buena correlación con titulación de la $\mathrm{SatO}_{2}<97 \%$, su carácter no invasivo y su seguimiento continuo en el paciente y muchas guías actualmente la recomiendan como método de elección ${ }^{(28)}$. En nuestro estudio la valoración se ajustó solo a los pacientes que tenían sistemas de alto flujo con medición exacta de $\mathrm{FiO}_{2}$ para evitar las estimaciones imprecisas de la $\mathrm{FiO}_{2}$ en cánulas nasales o máscaras oro-nasales de flujo libre de $\mathrm{O}_{2}$.

La gran mayoría de los pacientes ingresaron con oxigenoterapia convencional en cánula nasal o máscara. Uno de los aspectos que podría mejorar la situación de muchos pacientes en su admisión, sobre todo los trasladados desde distancias mayores, sería disponer en las 
unidades de traslados de otros sistemas de soporte respiratorio (CNAF o BIPAP) y capacitación de los recursos humanos que realizan los traslados de esta población de niños más graves.

Se observaron datos similares entre los hallazgos radiológicos en ambos grupos, predominando la hiperinsuflación y el patrón intersticial, como está descrito de forma clásica en las bronquiolitis. Solo se destaca el mayor porcentaje de descripción de consolidaciones en las UI (una cuarta parte de los pacientes), aunque el diagnóstico de probable sobreinfección bacteriana se planteó en menos del 3\% de los casos. Esta disociación clínico-radiológica es característica de la semiología de la bronquiolitis. En menos de 7\% de las radiografías fueron descritas atelectasias, hallazgo que se suele encontrar de forma frecuente en las bronquiolitis graves y moderadas. Estos datos son similares a los de otras series ${ }^{(9,21,22,27)}$.

En relación con la etiología, no hay diferencias entre ambas poblaciones, siguiendo el patrón clásico de predominio del VRS y un $30 \%$ de pacientes con estudio viral negativo, si bien excepcionalmente se emplearon otras técnicas que permiten identificación de otras etiologías virales además de influenza, VRS y adenovirus. Aunque la coinfección viral ha sido vinculada a un mayor riesgo de desarrollar cuadros más graves, menos de $3 \%$ presentaron etiología múltiple en la detección viral en el aspirado de secreciones. Aunque hay una mayor sospecha diagnóstica de sobreinfección bacteriana en las UM, solo se plantea en el $8 \%$ de los casos, ya que está descrita como un hecho relativamente infrecuente en la bronquiolitis $^{(9,21,22,28)}$.

A pesar de que las guías actuales excluyen las formas graves de bronquiolitis y no existen guías específicas para formas severas, éstas sugieren que no hay una evidencia clara para el uso de muchas de las terapéuticas utilizadas clásicamente, un porcentaje elevado de los pacientes han recibido al menos una de ellas en nuestro estudio. Esta falta de adherencia a guías de práctica clínica internacionales está descrita en varios trabajos ${ }^{(4,9,29)} \mathrm{y}$, además de elementos vinculados a la "resistencia al cambio" y al hecho de la frustración de no disponer de ningún tratamiento que se haya mostrado realmente efectivo y con impacto clínico significativo en un paciente que se deteriora clínicamente, hoy en día también está en discusión la existencia de diferentes tipos de fenotipos en la bronquiolitis que se asociarían a diferentes evoluciones y respuestas al tratamiento $^{(5)}$. Las actuales guías de la AAP, la AEP y las del NICE en Gran Bretaña desaconsejan el uso de broncodilatadores, adrenalina y corticoides en la bronquiolitis $^{(6,24,30,31)}$. Este hecho refleja el actual estado de incertidumbre y de variabilidad en la práctica clínica exis- tente en el tratamiento de la bronquiolitis y de la que no escaparon las UCIP nacionales analizadas, muchas veces amparados en la exclusión explícita que hacen estas guías para las formas graves.

Se destaca la utilización elevada de antibióticos (más del $60 \%$ ) y de suero salino hipertónico (35\%) en las UI respecto a las UM. Posiblemente este tipo de trabajo y la visualización de los datos de cada centro a través de una plataforma cooperativa, como la nuestra, permita a las diferentes UCIP comparar sus datos para realizar mejoras continuas de la calidad de atención para un uso racional de antibióticos en la bronquiolitis, aunque hay datos recientes que abogan por un uso más liberal de los antibióticos en las bronquiolitis que requirieron ventilación invasiva $^{(32)}$.

No existe un soporte respiratorio estandarizado en la bronquiolitis, pero sí un escalonamiento progresivo en función de la gravedad de la insuficiencia respiratoria y el tipo de alteración gasométrica del paciente. Hoy en día existe una discusión sobre el papel de los diferentes métodos no invasivos, aunque se puede considerar al CNAF como una primera opción de tratamiento. En este sentido, la aplicación de formas menos invasivas que eviten morbilidades mayores se ha ido imponiendo, extendiéndose el uso cada vez mayor de la oxigenoterapia de alto flujo (CNAF) y la ventilación no invasiva (VMNI) y el concepto cada vez más extendido en el cuidado intensivo pediátrico de que "menos es más" en la mayoría de las ocasiones ${ }^{(1,9,19,20,33-36)}$. En este sentido, las UM tienden a un mayor uso de la CNAF tanto al ingreso como en la evolución de sus pacientes, mientras que las UI más de $40 \%$ de los pacientes reciben algún soporte con presión positiva, precisando la VMI en casi un tercio de los casos con una relación 3:1 respecto al uso de VMI/VMNI o CPAP; esta proporción también se mantiene en las UM.

Si bien parte de esta diferencia se puede explicar por el hecho de tratarse de una población inicialmente con más gravedad en las UI respecto a las UM, la filosofía de estas redes colaborativas apunta a perfeccionar aspectos del tratamiento que en otras UCIP funcionan mejor y buscar causas mejorables que puedan incrementar el uso de modalidades menos invasivas en las UI. Datos de estudios observacionales en UCIP de varios países muestran porcentajes algo menores de uso de VMI, si bien en este hecho pueden influir otros factores relacionados con el diseño y estructura disponible para estas patologías $^{(9,33,34)}$.

El promedio de estadía no fue alto, estando dentro de los valores encontrados en la literatura. La mortalidad en esta serie fue baja, incluso comparada con la mortalidad calculada por PIM-3, con REM por debajo del 0,5, similar a la descrita en otras series ${ }^{(1,9,18,21,33)}$. La presencia de 
morbilidades fue similar a la informada en otros trabajos, siendo la presencia de síndrome de abstinencia y delirio los principales puntos a mejorar, existiendo hoy en día varias estrategias para minimizar estos aspectos en la población pediátrica más grave. Hubo una tasa muy baja de fuga aérea y de necesidad de tratamientos de rescate, lo que se puede traducir en una buena evolución global de ambas series. En esta línea, la aparición de nueva morbilidad es mínima en estas series, si bien es un problema que puede estar subestimado ${ }^{(36-38)}$.

Nuestro trabajo presenta algunas limitaciones. Al tratarse de un estudio poblacional que depende de la inclusión de datos por parte de cada centro, pueden existir diferencias ligadas a la interpretación y la inclusión de variables clínicas en relación con estudios con diseño más controlado, por ejemplo, la discrepancia de las definiciones clásicas de bronquiolitis y lo que el médico tratante puede catalogar como bronquiolitis sin usar una definición estricta, pero al ser una inclusión multicéntrica de un escenario sin definiciones rígidas, nos permite visualizar una realidad más ligada a la práctica real y que trasciende un solo centro.

\section{Conclusiones}

Este estudio poblacional pudo definir las principales características de los pacientes con diagnóstico clínico al ingreso de bronquiolitis (definida por el médico de urgencia tratante, como fue señalado) ingresadas en diferentes UCIP a escala nacional, detectando diferencias entre UI y UM. En las UI los pacientes presentan mayor gravedad y provienen en mayor proporción de otros centros más lejanos, con aspectos relacionados al tipo de soporte respiratorio utilizado. En las UM se realizó un soporte respiratorio menos invasivo, sin variación destacable en los resultados asistenciales finales.

Aunque algunos desestiman las guías de bronquiolitis en su forma grave por ser frecuentemente un criterio de exclusión inicial de las recomendaciones sin justificación clara de la razón, podemos decir que detectamos gran variabilidad en el uso de distintas intervenciones terapéuticas actualmente desaconsejadas por las guías de práctica clínica, como broncodilatadores, antibióti$\cos$ y corticoides, potencial indicador de sobre e infrauso de terapias, lo que indica un área de potencial mejoría.

La evolución de la cohorte tuvo una evolución favorable con una mortalidad baja. El conocer nuestros resultados, compartiéndolos y comparándolos con los de varios centros con distintos enfoques y prácticas, constituye un enriquecimiento de nuestra práctica y un motor de mejora en la atención que brindamos a los niños críticos de nuestro país.

\section{Summary}

Objectives: to describe the clinical and epidemiological characteristics of children admitted for bronchiolitis in 13 Pediatric Intensive Care Units (UCIP) in Uruguay and compare the final care outcomes between Montevideo (UM) and Interior of the country (IU).

Method: multicenter, retrospective, observational study of data entered in the LARed Network prospective database. Children over 1 month and younger than 2 years admitted between May 1, 2017 and April 30, 2019 with a diagnosis of Community Bronchiolitis were included in the study. Demographic and clinical data were analyzed, as well as interventions and discharge outcomes.

Results: 666 cases were analyzed. No significant differences in comorbidity and respiratory support were detected at admission. In IU patients were referred more frequently from another hospital. The distance and average time, as well as the percentage of transfers greater than $50 \mathrm{~km}$, was also higher. In IU, patients had greater clinical and gasometrical severity at admission. The radiological and etiological profile was similar (VRS at > $50 \%$ ). The overall indication of corticosteroids excee$\operatorname{ded} 25 \%$ and that of bronchodilators exceeded $85 \%$. The prescription for antibiotics and nebulized adrenaline was higher in IU. The high flow nasal cannula (HFNC) was globally the most widely used respiratory support method, although increased use of invasive mechanical ventilation (IMV) and CPAP in IU ( $43 \%$ vs $28 \%$ in UM) was observed. There were no differences in the number of complications from IVF or non-invasive ventilation, nor in the use of rescue therapies. There were also no significant differences in the length of stay at UCIP or in absolute and adjusted mortality and there was only one case of new morbidity.

Conclusions: children admitted to IU had higher severity scores and more transfer-related risk factors, received more antibiotics and invasive support. HFNC was the most widely used type of respiratory support in the country. A high prescription of non-recommended therapies such as bronchodilators and corticosteroids was detected. Mortality and complications were low, as were the generation of new morbidity.

\section{Resumo}

Objetivos: descrever as características clínicas e epidemiológicas de crianças internadas por bronquiolite em 13 Unidades de Terapia Intensiva Pediátrica (UTIP) do Uruguai e comparar os resultados finais do atendimento entre UTIP de Montevidéu (UM) e do Interior do país (IU). 
Material e métodos: estudo observacional retrospectivo multicêntrico dos dados inseridos no banco de dados prospectivo da Rede LARed. Foram incluídas crianças maiores de 1 mês e menores de 2 anos internadas no período de $1^{\circ}$ de maio de 2017 a 30 de abril de 2019 com diagnóstico de bronquiolite comunitária. Dados demográficos e clínicos, bem como intervenções e desfechos na alta, foram analisados.

Resultados: foram analisados 666 casos. Não foram detectadas diferenças significativas nas comorbidades ou no suporte respiratório na admissão. No IU, os pacientes foram encaminhados com maior frequência a outro hospital. A distância e o tempo médios, assim como o percentual de transferências superiores a $50 \mathrm{~km}$, também foram maiores. No IU, os pacientes apresentaram maior gravidade clínica e gasométrica na admissão. O perfil radiológico e etiológico foi semelhante. $\mathrm{O}$ vírus sincicial respiratório (RSV) foi isolado em $>50 \%$. A indicação global de corticosteroides ultrapassou $25 \%$ e a de broncodilatadores $85 \%$. A prescrição de antibióticos e adrenalina nebulizada foi maior no IU. A cânula nasal de alto fluxo (CNAF) foi o método de suporte respiratório mais utilizado, embora tenha sido observado um maior uso de ventilação mecânica invasiva (VMI) e CPAP no IU ( $47 \%$ vs $28 \%$ em UM). Não houve diferenças no número de complicações devido à VMI ou Ventilação Não Invasiva, ou no uso de terapias de resgate. Também não foram observadas diferenças significativas no tempo de internação na UTIP ou na mortalidade absoluta e ajustada, havendo apenas um caso de nova morbidade.

Conclusões: as crianças admitidas no IU apresentaram maior gravidade na admissão e mais fatores de risco relacionados ao mau prognóstico na transferência, recebendo mais antibióticos e suporte invasivo do que as internadas em UM. O CNAF foi o tipo de suporte respiratório mais utilizado no país. Detectou-se alto percentual de prescrição de terapias não recomendadas, como broncodilatadores e corticosteroides. A mortalidade e as complicações foram baixas, assim como a geração de morbidade residual.

\section{Bibliografía}

1. Machado K, Notejane M, Mello M, Pirez C, Giachetto G, Pérez W. Infecciones respiratorias agudas bajas en niños menores de 2 años. Hospitalizaciones durante el invierno del año 2014. Anfamed 2018; 5(1):82-103.

2. Harish N, Simoes E, Rudan I, Gessner B, Azziz-Baumgartner E, Zhang JSF. Global and regional burden of hospital admissions for severe acute lower respiratory infections in young children in 2010: a systematic analysis. Lancet 2013; 381(9875):1380-90.

3. Hancock DG, Charles-Britton B, Dixon DL, Forsyth KD. The heterogeneity of viral bronchiolitis: a lack of universal consensus definitions. Pediatr Pulmonol 2017: 52(9):1234-40. doi: 10.1002/ppul.23750.

4. Korppi M, Mecklin M, Heikkilä P. Review shows substantial variations in the use of medication for infant bronchiolitis between and within countries. Acta Paediatr 2019; 108(6):1016-22.

5. Rodríguez-Martínez C, Castro-Rodriguez J, Nino G, Midulla $\mathbf{F}$. The impact $\mathrm{f}$ viral bronchiolitis phenotyping: is time to consider phenotype-specific responses to individualize phamacological management?. Paediatr Respir Rev 2020; 34:53-8. doi: 10.1016/j.prrv.2019.04.003

6. Ralston SL, Lieberthal AS, Meissner HC, Alverson BK, Baley JE, Gadomski AM, et al. Clinical practice guideline: the diagnosis, management, and prevention of bronchiolitis. Pediatrics 2014; 134(5):e1474-502.

7. Martínez Blanco B, Martinón-Torres F. ¿Es urgente actualizar las guías de práctica clínica españolas para el tratamiento de la bronquiolitis aguda?. An Pediatr (Barc) 2016; 85(2):106-8.

8. Franklin D, Babl FE, Schlapbach LJ, Oakley E, Craig S, Neutze $\mathbf{J}$, et al. A randomized trial of high-flow oxygen therapy in infants with bronchiolitis. N Eng J Med 2018; 378(2):1121-31.

9. Dohna-Schwake C, Mücher K, Stehling F, Rothoeft T, Roll C, Brevis Nuñez F, et al. Differences for medical care for acute severe viral bronchiolitis in two urbans areas in Europe. Klin Padiatr 2018; 230:245-50.

10. González-Dambrauskas S, Díaz F, Carvajal C, Monteverde-Fernández N, Serra A. La Colaboración para mejorar los cuidados médicos de nuestros niños. El desarrollo de una Red Pediátrica Latinoamericana: LARed. Arch Pediatr Urug 2018; 89(3):194-202.

11. Harris P, Taylor R, Thielke R, col. Research electronic data capture (REDCap - a metadata-driven methodology and workflow process for providing translational research informatics support. J Biomed Inform 2009; 42(2):377-81.

12. Wang EE, Milner RA, Navas L, Maj H. Observer agreement for respiratory signs and oximetry in infants hospitalized with lower respiratory infection. Am Rev Respir Dis 1992; 145:106-9.

13. Liu LL, Gallaher MM, Davis RL, Rutter CM, Lewis TC, Marcuse EK. Use of a respiratory clinical score among different providers. Pediatr Pulmonol 2004; 37(3):243-8.

14. Wood DW, Downes JJ, Lecks HI. A clinical scoring system for the diagnosis of respiratory failure. Preliminary report on childhood status asthmaticus. Am J Dis Child 1972; 123: 227-8.

15. Pollack MM, Holubkov R, Glass P, Dean JM, Meert KL, Zimmerman J, et al. Functional Status Scale: a new pediatric outcome measure. Pediatrics 2009; 124(1):e18-28.

16. Moynihan K, McSharry B, Reed P, Dphil PR, Buckley D. Impact of retrieval, distance travelled and referral center on outcomes in unplanned admissions to a national ICU. Pediatr Crit Care Med 2016; 17:e34-42. 
17. Kawaguchi A, Saunders LD, Yasui Y, DeCaen A. Effects of medical transport on outcomes in children requiring intensive care. J Intensive Care Med 2018; 35(9):889-95. doi: $10.1177 / 0885066618796460$.

18. Gazhaly M, Nadel S. Characteristic of children admitted to intensive care with acute bronchiolitis. Eur J Pediatr 2018; 177:913-20.

19. Morosini F, Notejane M, Machado K, Paéz M, Rompani E, Taboada R, et al. Ventilación no invasiva y oxigenoterapia de alto flujo en salas de cuidados moderados. Experiencia en la Unidad de Cuidados Especiales Respiratorios Agudos del Hospital Pediátrico del CHPR durante 2013-2016. Arch Pediatr Urug 2018; 89(2):78-85.

20. Clayton J, McKee B, Slain CN, Rotta AT, Shein SL. Outcomes of children with bronchiolitis treated with high-flow nasal cannula or non-invasive pressure ventilation. Pediatr Crit Care Med 2019; 20(2):128-35.

21. Ramos-Fernández JM, Pedrero-Segura E, Gutiérrez-Bedmar M, Delgado-Martín B, Cordón-Martínez AM, Moreno-Pérez D, et al. Epidemiología de los ingresos por bronquiolitis en el sur de Europa: análisis de las epidemias 2010-2015. An Pediatr (Barc) 2017; 87(5):260-8.

22. Flores-González J, Valladares CM, Yun-Castilla C, Mayordomo-Colunga J, Quesada SP, Martín Delgado CM, et al. Association of fluid overload in critically ill children with bronchiolitis: bronquiolitis en la Unidad de Cuidados Intensivos Pediátricos (BRUCIP) Study. Pediatr Crit Care Med 2019; 20(3):e130-6.

23. Jacobs A, Flechet M, Vanhorebeek I, Verstraete S, Ingels C, Casaer MP, et al. Performance of pediatric mortality prediction scores for PICU mortality and 90-days mortality. Pediatr Crit Care Med 2019; 20(2):113-9.

24. Giachetto G, Vomero A, Pandolfo S, Notejane M, García L, Cavallieri F, et al. Validación del score clínico de Tal modificado para la evaluación de severidad en bronquiolitis. Arch Pediatr Urug 2018; 89(Suppl. 1):43-8.

25. Pérez W, Giachetto G, García L, Vomero A, Pandolfo S, Cavallieri F, et al. Opinión de expertos sobre el artículo "Validación del score clínico de Tal modificado para la evaluación de severidad en bronquiolitis". Arch Pediatr Urug 2019; 90(3):89-94.

26. Duarte-Dorado DM, Madero-Orostegui DS, Rodriguez-Martinez CE, Nino G. Validation of a scale to assess the severity of bronchiolitis in a population of hospitalized infants. J Asthma 2013; 50(10):1056-61. doi: 10.3109/02770903.2013.834504.

27. Serra JA, González-Dambrauskas S, Vásquez Hoyos P, Carvajal C, Donoso A, Cruces P, et al. Variabilidad terapéutica en lactantes con bronquiolitis hospitalizados en unidades de cuidados intensivos latinoamericanas. Rev Chil Pediatr 2020; 91(2):216-25. doi:10.32641/rchped.v91i2.1156.
28. Pilar Orive FJ, López Fernández Y; UCIP. Hospital de Cruces. Guía para el manejo de la bronquiolitis aguda. Sociedad y Fundación Española de Cuidados Intensivos Pediátricos, 2013. Disponible en: http://secip.com/wp-content/uploads/2018/06/Protocolo-Bronquiolitis-2013.pdf [Consulta: 6 junio 2019].

29. Oakley E, Brys T, Borland M, Neutze J, Philipps N, Krieser $\mathbf{D}$, et al. Medication use in infants admitted with bronchiolitis. Emerg Med Australas 2018; 30(3):389-97.

30. National Institute for Health and Care Excellence. Bronchiolitis in children: diagnosis and management. June 2015. Disponible en: http:/www.nice.org.uk/guidance/ng9 [Consulta: junio 2016].

31. Stobbelaar K, Kool M, de Kruijf D, Van Hoorenbeck K, Jorens P, De Doooy J, et al. Nebulised hypertonic saline in children with bronchiolitis admitted to the paediatric intensive care unit: a retrospective study. J Paediatr Child Health 2019; 55(9):1125-32. doi: 10.1111/jpc.1437.

32. Shein SL, Kong M, McKee E, O'Riordan MA, Toltzis P, Randolph AG. Antibiotic prescription in young children with respiratory syncytial virus-associated respiratory failure and associated outcomes. Pediatr Crit Care Med 2019; 20(2):101-9.

33. Morris JV, Kapetanstrataki M, Parslow RC, Davis PJ, Ramnarayan P. Patterns of use of heated humidified high-flow nasal cannula therapy in PICUs in the United Kingdom and Republic of Ireland. Pediatr Crit Care Med 2019; 20(3):223-32.

34. Metge P, Grimaldi C, Hassid S, Thomachot L, Loundou A, Martin C, et al. Comparison of high-flow humidified nasal cannula to nasal continuous with acute bronchiolitis: experience in a pediatric intensive care unit. Eur J Pediatr 2014; 173:953-8.

35. Milési C, Essouri S, Pouyal R, Liet JM, Afanetti M, Portefaix A, et al. High flow nasal cannula (HFNC) versus nasal continuous positive airway pressure (nCPAP) for the initial respiratory management of acute viral bronchiolitis in young infants: a multicenter randomized controlled trial (TRAMONTANE study). Intensive Care Med 2017; 43:209-16

36. Shein SL, Slain K, Wilson-Costello D, McKee B, Rotta AT. Temporal changes of prescription of neuropharmacological drugs and utilization of resources related to neurologic morbidity in mechanically ventilated children with bronchiolitis. Pediatr Crit Care Med 2017; 18(12):e606-14.

37. Horvat CM, Clark RS. Healthcare in the PICU may be more complicated than we thought-we knew? Pediatr Crit Care Med 2017; 18(12):1188-9.

38. Baudin F. Viral bronchiolitis in PICUs: looking further than the acute phase!. Pediatr Crit Care Med 2017; 18(12):1178-9. 


\section{Contribuciones de los autores}

Los doctores P. Vásquez-Hoyos, Franco Díaz-Rubio y Luis Martínez-Arroyo tuvieron acceso completo a todos los datos del estudio y asumen la responsabilidad de la integridad de los datos y la precisión del análisis de datos.

Concepto y diseño: Luis Martínez-Arroyo, P. Vásquez-Hoyos.

Adquisición. Análisis o interpretación de datos: P. Vásquez-Hoyos, Luis Martínez-Arroyo.

Redacción del manuscrito: Luis Martínez-Arroyo.

Revisión crítica del manuscrito de contenido intelectual importante: todos los autores.

Análisis estadístico: P. Vásquez-Hoyos, Luis Martínez-Arroyo.

Apoyo administrativo, técnico o material: P. Vásquez-Hoyos, Franco Díaz, Sebastián González-Dambrauskas.

Redacción de manuscritos: todos los autores.

Aprobación final del manuscrito: todos los autores.

Supervisión: P. Vásquez-Hoyos y S. González-Dambrauskas.

Luis Martínez-Arroyo, https://orcid.org/0000-0003-1243-2807

Franco Díaz-Rubio, https://orcid.org/0000-0003-4763-074X

Sebastián González-Dambrauskas, https://orcid.org/0000-0003-4775-227X

Nicolás Monteverde-Fernández, https://orcid.org/0000-0002-4734-1633

Alberto Serra, https://orcid.org/0000-0001-6264-4141

Luis Eduardo Pedrozo Ortiz, https://orcid.org/0000-0001-8534-1901

Lorena Soledad Menta Romano, https://orcid.org/0000-0002-6269-3477

Luis Castro, https://orcid.org/0000-0002-0200-4799

Pablo Vásquez-Hoyos, https://orcid.org/0000-0002-4892-5032 